\title{
Experiencias de educación para la salud en fisioterapia
}

\author{
Experiences of health education in physical therapy
}

Jacqueline Hernández-Sánchez ${ }^{*}$, Leidy Johanna Lozano-García², Yurley Andrea Murillo-Varela³

1 Enfermera, Maestría en Tecnología Educativa y Medios Innovadores de la Educación. Docente cátedra de la Escuela de Fisioterapia, Universidad Industrial de Santander. Bucaramanga, Colombia. e-mail: jacqueline.hernandez@correo.vis.edu.co

2 Fisioterapeuta. Clinica Carlos Ardila Lule, Clínica Foscal Internacional y Clinica de Urgencia Bucaramanga. Bucaramanga, Colombia e-mail: yurand16@hotmail.com

3 Fisioterapeuta. Unidad Médica Clinilago. Bucaramanga, Colombia. e-mail: yurand16@hotmail.com

Fecha de recepción: Septiembre 24 - 2015 Fecha de revisión: Mayo 27 - 2016 Fecha de aceptación: Diciembre 12 - 2016

Hernández-Sánchez J, Lozano-García LJ, Murillo-Varela YA. Experiencias de educación para la salud en fisioterapia. Univ. Salud. 2016;18(3):576-585. DOI: http://dx.doi.org/10.22267/rus.161803.63

\begin{abstract}
Resumen
Introducción: La educación para la salud es una herramienta fundamental en salud pública, donde los fisioterapeutas cumplen un rol importante; fomentando comportamientos que protegen la salud o previniendo aquellos que la ponen en riesgo. Objetivo: Identificar las características de experiencias en educación para la salud que llevan a cabo fisioterapeutas a nivel mundial. Materiales y métodos: Estudio descriptivo, mediante revisión documental de experiencias divulgadas en artículos publicados entre 1995 y 2014. Resultados: Se identificaron 30 experiencias de fisioterapeutas como educadores para la salud, reportadas en artículos científicos. Las áreas de aplicación fueron, musculo-esquelética $(20 ; 66,6 \%)$ y actividad física o ejercicio físico $(6 ; 20 \%)$. Existen debilidades en las bases teóricas; además, se utilizan principalmente estrategias metodológicas tradicionales como la consejería (24,2\%), la educación individual $(21,2 \%)$ y la combinación de consejería con material de educación y comunicación $(9,1 \%)$; los impresos son ampliamente usados. Las evaluaciones que se realizan son principalmente de tipo cuantitativo. Conclusiones: Son escasas las publicaciones científicas sobre intervenciones de educación para la salud realizadas por fisioterapeutas. Las reportadas en este artículo se desarrollan principalmente en el área músculo-esquelética, pero no se encuentra una clara fundamentación teórica; existiendo confusión y uso indiscriminado del término educación para la salud y promoción de la salud. Se plantean retos normativos y formativos para los fisioterapeutas como educadores para la salud, así como problemas para ser resueltos en futuras investigaciones.
\end{abstract}

Palabras clave: Educación para la salud; fisioterapia; estilos de vida; promoción de la salud. (Fuente: DeCS, Bireme).

\begin{abstract}
Introduction: Health education is a fundamental tool in public health, where the physiotherapists play an important role by either promoting behaviors that protect the health or preventing those that put it at risk. Objective: To identify the characteristics of experiences of HE that physiotherapists carry out worldwide. Materials and methods: A descriptive study was performed by using documentary review of experiences reported in articles published from 1995 to 2014. Results: 30 experiences of physiotherapists as health educators were identified, which were reported in scientific papers. The areas of application were musculoskeletal $(20 ; 69 \%)$ and physical activity or exercise (6; $20.6 \%$ ). There are weaknesses in the theoretical foundations; in addition, traditional methodological strategies such as counseling $(24.2 \%)$, individual education $(21.2 \%)$ and the combination of counseling with educational material
\end{abstract}


(9.1\%) are used. Printed materials are also widely used. Evaluations carried out are mainly quantitative. Conclusions: There are few scientific publications of experiences about Health education in physiotherapy. Those reported in this article are mainly developed in the muscle-skeletal area. However, there is not a clear theoretical foundation; there is confusion and an indiscriminate use of the term Health education and health promotion. There are normative and training challenges for physiotherapists as health educators; as well as problems to be solved in future research.

Keywords: Health education; physiotherapy; lifestyles; health promotion. (Source: DeCS, Bireme).

\section{Introducción}

El desarrollo de las aptitudes personales es una de las líneas de acción de la Promoción de la Salud (PS), planteadas desde la Primera Conferencia Internacional en Ottawa en 19861, estableciendo los estilos de vida como factor determinante de la salud individual, grupal y colectiva; aunque desde 1978 en la Declaración de Alma Ata², ya se incluía la educación como una estrategia para la prevención y lucha de los principales problemas de salud, así como para desarrollar capacidad en las comunidades para participar. Es así como desde hace tiempo, las actividades educativas se han venido realizando con el fin de prevenir aquellas conductas que representan riesgo o fomentar las que requiere la persona en el proceso de tratamiento de su enfermedad o rehabilitación; por lo que se constituye en una de las acciones claves en salud pública ${ }^{3}$.

La práctica educativa por parte de los profesionales de la salud, generalmente ha representado un reto debido a los múltiples factores que acarrea el compromiso de intervenir el comportamiento de las personas; pues como se sabe, el término educación es muy amplio; tiene sus raíces en el latín educhere, que significa "sacar, extraer" o de educare: "formar, instruir" 4 . En concreto, la educación se puede definir como el proceso multifactorial en el cual se desarrollan conocimientos, valores, costumbres, que pueden conllevar a cambios en el comportamiento del ser humano y no se rige a un aula o a una institución, puesto que se está dando constantemente mediante experiencias, acciones, actitudes; es decir, la educación no se concentra sólo en proceso de intervención por parte de un educador, docente o pedagogo ${ }^{4}$.
Aplicada entonces la educación en el contexto de la salud de las personas, su objetivo principal, según la Organización Mundial de la Salud (OMS)5, no sólo es el de aumentar el conocimiento sobre el comportamiento de salud personal, sino también desarrollar habilidades que demuestren la viabilidad política y las posibilidades organizativas de diversas formas de acción para abordar los determinantes sociales, económicos y ambientales de la salud. Su principal finalidad es fomentar estilos de vida saludables o prevenir aquellos que representan un riesgo, es decir que interviene los comportamientos relacionados con la salud; tiene sentido positivo de ayuda y potenciación de la capacidad de la persona para la participación y gestión de su propia salud y poder desarrollarse en un proceso de salud integral y de calidad de vida 5 .

Una de las definiciones de Educación para la Salud (EpS) que más ha sido aceptada, es la propuesta por la OMS, quien la concibe como las oportunidades de aprendizaje creadas conscientemente que suponen una forma de comunicación destinada a mejorar la alfabetización en salud, incluida la mejora del conocimiento de la población en relación con la salud y el desarrollo de habilidades personales que conduzcan a la salud individual y de la comunidad6. Esta definición se ha venido retomando a través de los años, pero en esencia no ha cambiado, puesto que se enfatiza en el aprendizaje para el desarrollo de saberes, actitudes y habilidades necesarias para mantenerse saludable o prevenir la enfermedad.

Se reconoce que los estilos de vida, es decir el conjunto de comportamientos que caracterizan la vida de una persona o de un grupo de personas, es un factor que junto con las condiciones de vida (el nivel educativo, las condiciones laborales, el 
hambre, las desigualdades sociales, los ingresos, el medio ambiente, entre otras), son los que determinan su salud 1,6,7. Los estilos de vida se basan en patrones de comportamiento identificables, determinados por la interacción entre las características personales individuales, las interacciones sociales y las condiciones de vida socioeconómica y ambientales ${ }^{1}$. Una definición similar es la que presentan Bibeau et al., citado por Anaya, quienes consideran los estilos de vida como una dimensión colectiva y social, que además comprende tres aspectos interrelacionados: el material, el social y el ideológico ${ }^{8}$.

Existen factores que inciden de manera directa o indirecta en los comportamientos relacionados con la salud, dentro de los que se encuentran los socioculturales y personales ${ }^{3}$. Adicionalmente, se han propuesto diversas teorías y modelos ${ }^{5,9,10}$ que tratan de explicar la naturaleza de las variables que intervienen en el comportamiento humano, en la literatura generalmente emergen tres categorías en las que se pueden organizar todos ellas, a saber: intrapersonales, interpersonales y comunitarios. Un ejemplo de cada una es, el Modelo Trasteórico o de Etapas de Cambio, la Teoría Cognitiva Social y el Modelo Ecológico, respectivamente.

Por otra parte, desde una perspectiva teórica, el rol del fisioterapeuta en el marco de la salud pública actual ha estado enfocado hacia diversos campos donde no sólo prima lo clínico, sino también la atención desde la planeación de programas y proyectos que brindan beneficios a la comunidad. Sin embargo, su práctica profesional es limitada. Así lo muestran revisiones realizadas a nivel internacional ${ }^{11}$, nacional ${ }^{12}$ y local ${ }^{13}$, donde se ha llegado a conclusiones como:

a) Se observa una evolución conceptual que trasciende de lo biológico hacia enfoques sociales, interdisciplinarios y complejos; sin embargo, en las intervenciones aún se evidencia predominio del modelo epidemiológico. Lo anterior, puede asociarse con que profesiones jóvenes como la fisioterapia que aunque surgió con un enfoque netamente rehabilitador se ha preocupado por ampliar sus campos de ejercicio profesional logrando participar en acciones de salud pública, pero esta participación aún es incipiente, por cuanto no logra reflejarse en la práctica de una manera contundente.

b) Se puede evidenciar que los profesionales no han dado cuenta en su práctica profesional de todas sus áreas de desempeño y campos de actuación para las que fue reglamentada.

c) Al identificar las acciones del fisioterapeuta en torno a la salud de los individuos y la comunidad, sus actuaciones se limitan a la intervención y rehabilitación de patologías acordes al quehacer clínico del profesional, dejando de lado las acciones propiamente dichas de promoción de la salud y prevención primaria de la enfermedad.

En los últimos años, dicho rol está enfrentando retos importantes a raíz de las nuevas estrategias y modelos de atención que han surgido producto de las necesidades de salud de las personas ${ }^{13}$. Por ello, la perspectiva de acción de este profesional se ha ampliado hacia la concepción del individuo dentro de un entorno específico que les ofrezca un ambiente saludable y les permita gozar de calidad de vida.

Es así como las situaciones de salud pública del momento, como es el aumento de las enfermedades crónicas no transmisibles (el cáncer, las enfermedades cardíacas, respiratorias y la diabetes $)^{14}$, plantean escenarios en donde la fisioterapia tiene aportes importantes por realizar; algunos de estos escenarios son los relacionados con los factores del ambiente, los estilos de vida, los riesgos laborales y el acceso a los servicios de salud, donde la EpS se requiere ${ }^{15-}$ 17. En otras palabras, este profesional está llamado a participar en los campos de la PS y la prevención primaria de la enfermedad.

En congruencia con lo anterior, la Confederación Mundial de Fisioterapia (en inglés, World Confederation Physical Therapy-WCPT) reconoce que el desempeño del fisioterapeuta puede darse en diferentes ámbitos como: clínicas, hospitales, escuelas, empresas, entre otros ${ }^{18}$. Igualmente, la Asociación Americana de Fisioterapeutas (en 
inglés, American Physical Therapy AsociationAPTA), determina que una de las acciones de este profesional es proporcionar servicios de prevención de la enfermedad y PS en el marco de su objeto de estudio: el movimiento corporal humano ${ }^{19}$.

En cuanto al rol del fisioterapeuta como educador para la salud, dentro de los lineamientos de la WCPT, se establece que en su práctica integral este profesional educa y ofrece consultas a los consumidores, el público en general, las organizaciones comunitarias, clubes y asociaciones en relación con los propósitos y beneficios de la terapia física, y el papel del fisioterapeuta y otro personal de apoyo ${ }^{18}$.

A pesar de la relevancia que tiene la educación en el campo de la salud, y particularmente, como parte del desempeño profesional del fisioterapeuta, autores como Alexander et al., refieren en su publicación que no se ha determinado con claridad qué áreas, estrategias metodológicas, conceptos y modelos utilizan los fisioterapeutas al momento de realizar la $\mathrm{EpS}^{20}$.

A partir de lo anterior, para esta revisión se planteó como objetivo identificar las características de experiencias de educación para la salud, realizadas por profesionales de fisioterapia en el entorno mundial. Los resultados de este trabajo aportan al planteamiento de investigaciones que profundicen acerca del rol del fisioterapeuta como educador para la salud, así como a identificar cuáles lineamientos son necesarios mejorar, fortalecer o establecer para apoyar la actuación profesional de fisioterapia en el uso de la EpS en la salud pública.

\section{Materiales y métodos}

Se llevó a cabo un estudio descriptivo, mediante revisión documental ${ }^{21}$ de experiencias en el ámbito mundial de fisioterapeutas como educadores para salud, reportadas en artículos científicos. Los criterios de inclusión fueron, que las publicaciones dieran cuenta acerca de este tipo de experiencias y que su publicación se hubiera realizado en el período de 1995 hasta el año 2014. Para la búsqueda de los artículos se utilizaron las siguientes palabras clave, tanto en español, portugués e inglés: fisioterapeuta, fisioterapia, comportamientos saludables, promoción de la salud, prevención de la enfermedad, modelo educativo, teorías del comportamiento, educación para la salud, estilos de vida, comportamientos de riesgo; utilizando AND/OR como operadores booleanos.

Las bases de datos consultadas fueron: CRCnetbase, E-libro, Ebrary, Ebsco MedicLatina, Ebsco Omnilife Full Text Mega, EBsco Readers`Guide Full Text Mega, ProQuest Medical Library, ProQuest Medline, Elsevier y Dynamed.

Una vez seleccionado el artículo que cumpliera con los criterios de inclusión antes mencionados, se capturó la información en una ficha de registro elaborada para este fin, en la cual se especificaron los siguientes datos: título del artículo, autores, área de desarrollo de la experiencia, conceptos en torno a la EpS, aplicación de un modelo o teoría, estrategia metodológica de la intervención educativa, tipo de evaluación realizada y resultados de la misma. Después de diligenciadas las fichas, por parte del equipo investigador, se llevó a cabo una revisión detallada y finalmente se extrajeron las características de las experiencias halladas, realizando la descripción de las mismas. Los resultados se analizaron utilizando estadística descriptiva. Este estudio fue avalado por el Comité de Investigación de la Escuela de Fisioterapia de la Universidad Industrial de Santander, Bucaramanga, Colombia.

\section{Resultados}

Se hallaron 30 experiencias de $\mathrm{EpS}^{22-52}$ en fisioterapia, reportadas en artículos que cumplieron con los criterios de inclusión.

\section{Características generales}

Los 30 artículos, producto de investigaciones originales, fueron publicados en el período de 1995-2011, reportan experiencias de EpS realizadas únicamente por profesionales de fisioterapia o con participación de ellos como parte del equipo de salud responsable de la 
intervención. Estos trabajos fueron realizados en países, como Australia, Inglaterra, Estados Unidos, Finlandia, Holanda, Brasil, España, China y Suecia. Entre los años 2004 a 2010 se incrementó este tipo de publicaciones. Las áreas en las que se realizaron estas intervenciones educativas fueron principalmente: musculoesquelética $(20 ; 66,6 \%)$ y actividad física o ejercicio físico $(6 ; 20 \%)$.

La mayoría de las intervenciones educativas tuvieron como público objetivo a personas adultas mayores de 18 años, aunque en muchas de ellas no especifican la edad, pues usan con frecuencia el término pacientes. En tres de los estudios los participantes fueron fisioterapeutas o estudiantes de fisioterapia, donde se evaluó su actuación como educadores; en los demás, la muestra de estudio estuvo integrada por usuarios de los servicios y los programas.

\section{Concepto de EpS y fundamentación teórica}

En cuanto al concepto de EpS asumido en las diferentes experiencias educativas, se encontró que el $86,7 \%(26)$ no lo describen y solamente en el 13,3\%(4) de las publicaciones revisadas hacen referencia a dicho concepto. En tres de estos últimos exponen de manera explícita que la EpS es una experiencia de aprendizaje orientada al cambio de comportamientos; pero en uno de los artículos de Kerssens y colaboradores, no se define como tal, solamente se hace referencia al deber ser de la EpS, así: "la educación debe adaptarse a las necesidades y estar en sintonía con la situación del paciente"27 (Tabla 1).

Tabla 1. Experiencias que incluyen el concepto de educación para la salud (EpS)

\begin{tabular}{|c|c|}
\hline Concepto de EpS & Autores \\
\hline $\begin{array}{l}\text { La educación debe adaptarse a las necesidades y en sintonía con la situación del paciente. } \\
\text { La educación de los pacientes es una experiencia de aprendizaje planeado que utiliza una } \\
\text { combinación de métodos tales como la enseñanza, la consejería y técnicas de modificación del } \\
\text { comportamiento, que influyen en los comportamientos en salud y conocimientos de los } \\
\text { pacientes. }\end{array}$ & $\begin{array}{l}\text { Kerssens et al, } 199927 \\
\text { Kerssens et al, } 199951\end{array}$ \\
\hline $\begin{array}{l}\text { Es un tipo de educación que busca modificar los conocimientos, actitudes y comportamientos } \\
\text { relacionados con la salud de los individuos, grupos y colectividades (...). Es un instrumento que } \\
\text { tienen los profesionales para conseguir la capacidad de controlar, mejorar y tomar decisiones } \\
\text { respecto a la salud o enfermedad de la población. }\end{array}$ & Rueda et al, $2011^{50}$. \\
\hline $\begin{array}{l}\text { Es un proceso dinámico, en el que las personas consideran la salud como un valor, incentivando } \\
\text { el uso de los servicios de salud, así como para obtener salud a través de sus propios esfuerzos y } \\
\text { acciones (...). Corresponde a una fracción de las actividades técnicas encaminadas a promover } \\
\text { la salud (...). Puede ser entendida como cualquier actividad de aprendizaje para alcanzar la salud } \\
\text { (...). No debe confundirse con información en salud, tiene como objetivo provocar cambios de } \\
\text { comportamiento individual, mientras que la Promoción de la Salud (que siempre incluye la EpS) } \\
\text { tiene el propósito de promover cambios en el comportamiento organizacional, capaz de } \\
\text { beneficiar la salud de la población. }\end{array}$ & Finkler \& Alvarenga, $2005^{39}$ \\
\hline
\end{tabular}

Por otra parte, se pudo establecer que en el artículo de Rueda et al., es claramente identificable el uso indiscriminado del concepto de promoción de la salud con el de EpS, el cual fue retomado de otros autores, se asume el primer concepto como "capacitar a la población para que pueda adoptar formas de vida saludables" ${ }^{50}$. En relación con la fundamentación teórica sobre el cambio o adopción de comportamientos en la que se sustentan las intervenciones educativas realizadas por los fisioterapeutas, los hallazgos muestran que no es frecuente que se mencione esta, aunque en el 23,3\% (7) se hace referencia a la Teoría Cognitiva Conductual, el 13,3\% (4) se basó en el Modelo Transteórico del Cambio de Prochaska y Diclemente y el 10\% (3) utilizó la Teoría Cognitiva Social de Bandura y en una experiencia se menciona que aplicaron el Modelo Biopsicosocial de Engel. 
Estrategias metodológicas aplicadas en la EpS En las experiencias educativas revisadas, se encontró que las estrategias metodológicas más utilizadas fueron: la consejería $(24,2 \%)$, la educación individual $(21,2 \%)$ y la combinación de consejería con material de educación y comunicación $(9,1 \%)$ (Tabla 2). El uso del material educativo impreso es frecuentemente incluido como ayuda educativa.

\section{Tabla 2. Estrategias metodológicas aplicadas en las intervenciones de EpS}

\begin{tabular}{|c|c|c|}
\hline Estrategia & $\mathbf{n}$ & $\%$ \\
\hline Educación individual & 7 & 21,2 \\
\hline Educación grupal & 2 & 6,1 \\
\hline Consejería & 8 & 24,2 \\
\hline Consejería más material impreso & 3 & 9,1 \\
\hline Educación grupal más material impreso & 1 & 3,1 \\
\hline $\begin{array}{l}\text { Educación individual más material } \\
\text { impreso }\end{array}$ & 1 & 3,1 \\
\hline $\begin{array}{l}\text { Educación grupal más educación } \\
\text { individual }\end{array}$ & 2 & 6,1 \\
\hline $\begin{array}{l}\text { Consejería más material audiovisual más } \\
\text { material educativo }\end{array}$ & 1 & 3,1 \\
\hline Consejería grupal más material impreso & 2 & 6,1 \\
\hline $\begin{array}{l}\text { Material audiovisual más material } \\
\text { educativo }\end{array}$ & 1 & 3,1 \\
\hline Auto-instrucción & 2 & 6,1 \\
\hline Material educativo impreso & 1 & 3,1 \\
\hline Video más consejería & 1 & 3,1 \\
\hline Consejería más llamadas telefónicas & 1 & 3,1 \\
\hline Total & 33 & 100 \\
\hline
\end{tabular}

\section{Evaluación de las intervenciones de EpS}

El enfoque más aplicado para realizar las evaluaciones fue el método cuantitativo (86,7\%; 26). Existe diversidad de diseños de los estudios aplicados, dentro de los que se destacan: estudios cuasi-experimentales, ensayos clínicos controlados, estudios transversales, entre otros. Las evaluaciones cualitativas solamente se identificaron en $4(13,3 \%)$ de las experiencias. Se reporta en $20(66,6 \%)$ intervenciones educativas, resultados positivos en términos de efectividad e impacto.

\section{Discusión}

Este estudio se logró identificar 30 intervenciones de EpS con participación de fisioterapeutas, en un período de 20 años (1995-2014); es posible que existan muchas más experiencias, algunas de ellas sin publicar. Las que se reportan en este artículo están principalmente relacionadas con dos de las áreas del campo de acción profesional, como son el área musculo-esquelética y la actividad física/ejercicio físico. Llama la atención que no es tan amplia la aplicación de la EpS, siendo tan diverso y extenso el objeto de estudio de fisioterapia (el movimiento corporal humano), tal como lo establece la Confederación Mundial de Fisioterapia (en inglés, WCPT), quien refiere que la terapia física ofrece servicios a individuos y poblaciones para desarrollar, mantener y restaurar el movimiento máximo y la capacidad funcional durante toda la vida; esto incluye la prestación de servicios en circunstancias donde el movimiento y la función están amenazados por el envejecimiento, la lesión, el dolor, las enfermedades, los trastornos y las condiciones o factores ambientales ${ }^{18}$.

La práctica de la fisioterapia se ocupa de la promoción de la salud, la prevención de la enfermedad, el tratamiento/intervención, habilitación o rehabilitación; es decir que sus alcances no se limitan a la atención al paciente o cliente directo, sino que también incluye, entre otros al desarrollo de otras estrategias de salud pública. Además, una parte integral de la terapia física es la interacción entre el fisioterapeuta y el paciente/cliente/familia o cuidador para desarrollar un entendimiento mutuo; en este tipo de interacción es necesaria para cambiar las conductas positivamente, el conocimiento del cuerpo y del movimiento, que pueden promover la salud y el bienestar 52,53 .

En el presente estudio se refleja que gran parte de las experiencias de EpS revisadas, no tienen en cuenta para su fundamentación los modelos ni las teorías que explican el cambio o adopción de comportamientos ${ }^{54}$ y los pocos que sí las utilizan, se basan principalmente en la Teoría Cognitiva Conductual.

Alexander et al., reportan en su trabajo que el $38 \%$ de los estudios que revisaron tuvieron en cuenta el Modelo Transteórico, fundamentación que fue también reportada en cuatro de las 
experiencias de la presente investigación. La importancia que tienen tanto los modelos como las teorías en EpS radica principalmente en que éstas ayudan a comprender los comportamientos, así como a diseñar las intervenciones educativas y a evaluarlas ${ }^{20}$.

Otro aspecto que es importante destacar como debilidad, es el que tiene relación con la fundamentación del concepto de EpS, pues el $82,8 \%$ de las intervenciones no lo describen. Este es un constructo fundamental para determinar desde qué enfoque se están asumiendo estas intervenciones educativas; el sentido que tenga la EpS va a determinar el para qué y el cómo de las mismas. Otro factor que influye es la tergiversación de dicho concepto, cuando se asume como sinónimo de la promoción de la salud, porque, aunque sí bien existe una estrecha relación entre las dos, ellas tienen un sentido y significado diferentes ${ }^{55}$.

En cuanto a las estrategias metodológicas aplicadas por los fisioterapeutas, se pudo establecer que se concentran en la aplicación de métodos de tipo individual, como es la consejería, la educación a la persona; además es frecuente el uso de materiales educativos tradicionales, como son los impresos (Tabla 2). Alexander et al., refieren en su trabajo que los folletos y volantes son uno de los medios educativos más usados, seguidos de los materiales audio-visuales ${ }^{16}$; la estructura de sesión más frecuente fue el consejo breve y la discusión; la técnica más utilizada fue las indicaciones motivacionales ${ }^{20}$. Por otra parte, esta situación denota el desconocimiento que se tiene sobre la poca efectividad que tienen los impresos, pues estudios como los de Giguère et al. y Farmer et al., han llegado a la conclusión que, si solo se utiliza este tipo de material, puede tener algún efecto en los resultados del proceso, pero no en los resultados del paciente, además no existe suficiente información para estimar de una forma fiable sus efectos en los resultados del tratamiento y su efectividad es aún incierta ${ }^{56,57}$.

También es notoria la falta de diversidad e innovación en las metodologías utilizadas, por ejemplo, no se encontró en ninguna de las experiencias, la integración de Tecnologías de
Información y Comunicación (TIC), las cuales en la actualidad tienen avances en su desarrollo y aplicación, tal como lo establece la reciente estrategia de la $\mathrm{OMS}^{58}$.

Además, se reportó resultados favorables para el cumplimiento de los objetivos en las evaluaciones de las intervenciones educativas. Es importante destacar como fortaleza de estas experiencias, que se realiza este tipo de evaluación, buscando establecer la efectividad y el impacto de las acciones educativas realizadas, contrario al estudio de Alexander et al., donde encontró que sólo en tres investigaciones hicieron valoraciones antes y después y solamente una de estas tres hizo seguimiento ${ }^{20}$.

\section{Conclusiones}

Existe una escasa publicación de artículos científicos sobre intervenciones de educación para la salud realizadas por fisioterapeutas. En las publicaciones que se encontraron, las áreas musculo-esquelética, actividad física y salud laboral son en las que más se reportan intervenciones educativas lideradas por estos profesionales. Además, es limitada la aplicación de las teorías y modelos de cambio o adopción de comportamientos en salud y existe una pobre diversidad en las estrategias metodológicas en las que se apoyan para la EpS, así como debilidades en las evaluaciones realizadas.

Existe un uso indiscriminado de los conceptos de EpS y Promoción de la Salud, lo cual amerita la necesidad de ampliar la fundamentación teórica sobre estos temas y establecer con claridad la relación que existe entre ellos; igualmente se hace necesario el desarrollo de competencias en los profesionales de fisioterapia, para que no solamente encuentren el sentido de lo que significa educar para la salud, sino que apliquen metodologías apropiadas para acompañar a las personas en el aprendizaje de comportamientos que protegen y fomentan su salud, así como en la evaluación de este tipo de intervenciones.

El papel del fisioterapeuta en la actualidad va más allá de la parte asistencial, exigiéndole mayor contribución en el logro de los objetivos de la PS y 
la prevención primaria de la enfermedad, para lo cual se hace necesario que tenga una mirada integral de las personas en los diferentes entornos donde viven, así como participando de un trabajo interdisciplinario e intersectorial que aporte a las transformaciones socioculturales, económicas y políticas. En este contexto, su rol como educador para la salud no es una opción, es un deber que le corresponde asumir. Por lo tanto, se hace necesario frente a este rol, una discusión profunda y lineamientos más específicos, por parte de la WCPT y la APTA, así como en las agremiaciones y la legislación que reglamenta la profesión en cada país.

Futuras investigaciones se pueden orientar a evaluar las intervenciones de EpS realizadas por fisioterapeutas, aplicando no solamente el método cuantitativo, sino también el cualitativo; inclusive estudios mixtos pueden ayudar a obtener una mirada amplia del fenómeno de estudio. Además, estudios sobre la evaluación de innovadoras metodologías educativas donde se apliquen las nuevas TIC, serían una contribución valiosa para diversificar la forma como educan los profesionales de fisioterapia.

\section{Agradecimientos}

Agradecimientos a los fisioterapeutas Angélica Patricia Toloza Pinzón, Leonel Sánchez Gómez y Wilson Cardozo Blanco, por sus aportes en la búsqueda bibliográfica.

\section{Conflicto de intereses}

Los autores declaran no tener ningún conflicto de intereses.

\section{Referencias}

1. Organización Mundial de la Salud. Carta de Ottawa. Primera Conferencia Internacional de Promoción de la Salud. Ottawa, Canadá. 1986.

2. Organización Panamericana de la Salud. Declaración de Alma-Ata. Conferencia Internacional sobre Atención Primaria de Salud. Alma-Ata, URSS. 1978.

3. Perea R. Promoción y educación para la salud. Tendencias innovadoras. Madrid: Díaz de Santos; 2009.

4. Educación. Diccionario etimológico. [última actualización 4 abril de 3013, a las 17:17.http://www.elcastellano.org/palabra.php. [acceso 2013-04-04].

5. World Health Organization. Health education: Theoretical concepts, effective strategies and core competencies: a foundation document to guide capacity development of health educators. Regional Office Forthe Eastern Mediterranean; 2012.

6. Organización Mundial de la Salud. Glosario de Promoción de la Salud [Internet] 1998 [acceso 4 de marzo de 2013] Disponible en: http://apps.who.int/iris/bitstream/10665/67246/1/ WHO_HPR_HEP_98.1_spa.pdf

7. Wilkinson R, Marmot M. Social determinants of health. The solid facts. Copenhagen: Word Health Organization; 2003.

8. Anaya L. Los estilos de vida saludables: Componente de la calidad de vida [Internet] 2001 [acceso 4 de abril de 2013] Disponible en: http://www.funlibre.org/documentos/lemaya1.htm.

9. Rimer B, Glanz K. Theory at a glance. A guide for health promotion practice, $2^{\text {nd }}$ ed. Bethesda, Maryland: Department of Health and Human Services; 2005.

10. Bandura A. Social learning theory. Englewood Cliff, NJ: Prentice-Hall; 1977.

11. Castro LE, Rodríguez YL. Tendencias epistemológicas de las acciones de la salud pública. Una revisión desde la fisioterapia. Rev. Fac. Nac. Salud Pública. 2015;33(2): 239-251. doi: 10.17533/udea.rfnsp.v33n2a11

12. Agredo V, Franco JA, Mina EP. Perfil profesional y ocupacional de los fisioterapeutas en Colombia. Revista CES Movimiento y Salud. 2015; 3(1): 35-43.

13. Cerpa J. Análisis de las acciones del fisioterapeuta en la ejecución de la política de salud pública en el Hospital de Suba. Informe final. Bogotá D.C: Escuela Superior de Administración Pública; 2010.

14. World Health Organization. Global Status Report on noncommunicable diseases 2014. Geneva: World Health Organization; 2014.

15. República de Colombia. Ministerio de la Protección Social. Guía de atención integral basada en la evidencia para dolor lumbar y enfermedad discal relacionados con la manipulación manual de cargas y otros factores de riesgo en el lugar de trabajo. Bogotá: Ministerio de la Protección Social; 2006.

16. República de Colombia. Ministerio de la Protección Social. Guía de atención integral basada en la evidencia para hombro doloroso relacionado con factores de riesgo en el trabajo. Bogotá: Ministerio de la Protección Social; 2006.

17. República de Colombia. Ministerio de la Protección Social. Guía de atención integral basada en la evidencia para desórdenes músculo-esqueléticos relacionados con movimientos repetitivos de miembros superiores. Bogotá: Ministerio de la Protección Social; 2006.

18. World Confederation for Physical Therapy. Guideline for standards of physical therapy practice. [última actualización 18 de octubre de 2011]. [acceso 3 de marzo de 2013] Disponible en: http://www.wcpt.org/sites/wcpt.org/files/files/Guide line_standards_practice_complete.pdf.

19. American Physical Therapy Association. Standards of practice for physical therapy.[última actualización 27 de enero de 2012]. [acceso 3 marzo de 2013] Disponible en: http://www.apta.org/uploadedFiles/APTAorg/About_ 
Us/Policies/BOD/Practice/CriteriaforStandardsofPract ice.pdf.

20. Alexander J, Bambury E, Mendoza A, Reynolds J, Veronneau R, Dean E. Health education strategies used by physical therapists to promote behaviour change in people with lifestyle-related conditions: A systematic review. Hong Kong Physiother J. 2012; 30:57-75.

21. Hernandez-Sampieri R, Fernández C, Baptista $P$. Metodología de la investigación. México D.F: Mc Graw Hill; 2014.

22. Kim P, Hayden J, Mior S. The cost-effectiveness of a back education program for firefighters: a case study. J Can Chiropr Assoc ,2004; 48(1):13-19.

23. Mayorga MJ, Fernández I, Bullón F, Morales C, Herrera J, Echevarría M. Impacto de un programa de educación sanitaria en pacientes con fibromialgia. Rev. Soc Esp Dolor. 2010;17(5):227-232.

24. Pakkala I, Read S, Leinonen R, Hirvensalo M, Lintunen T, Rantanen T. The effects of physical activity counseling on mood among 75- to 81-year-old people: A randomized controlled trial. Preventive Medicine. 2008; 46: 412-418.

25. Frost H, Lamb SF, Doll HA, Taffe P, Stewart-Brown S. Randomised controlled trial of physiotherapy compared with advice for low back pain. BMJ. 2004 doi:10.1136/bmj.38216.868808.7C

26. Klaber J, Jackson D, Richmond S, Hahn S, Coulton S, Farrin A, Manca A, Torgerson D. Randomised trial of a brief physiotherapy intervention compared with usual physiotherapy for neck pain patients: outcomes and $\begin{array}{lll}\text { patients' } & \text { preference. } & \text { BMJ. }\end{array}$ doi:10.1136/bmj.38286.493206.82

27. Kerssens J, Sluijs E, Verhaak P, Knibbe H, Hermans I. Back care instructions in physical therapy: a trend analysis of individualized back care programs. Phys Ther. 1999; 79:286-95.

28. De Vitta A, Bertaglia RS, Padovani CR. Efeitos de dois procedimentos educacionais sobre os sintomas musculoesqueléticos em trabalhadores administrativos. Rev Bras Fisioter, São Carlos. 2008; 12(1): 20-5.

29. Sheedy J, Smith B, Bauman A, Barnett A, Calderan A, Culbert $J$ and Jacka. A controlled trial of behavioural education to promote exercise among physiotherapy outpatients. Australian Journal of Physiotherapy. 2000; 46: 281-289.

30. Tipping C, Scholesb R, Coxb N. A qualitative study of physiotherapy education for parents of toddlers with cystic fibrosis. Journal of Cystic Fibrosis. 2010; 9: 205211.

31. Echsner J, Domholdt D. Amount of patient education in physical therapy practice and perceived effects. Phys Ther. 1996; 76:1089-1096.

32. Linton S, Andersson T. Can chronic disability be prevented? A randomized trial of a cognitive-behavior intervention and two forms of information for patients with spinal pain. Spine. 2000; 25(21): 2825-31.

33. Brown C, Gottschalk M, Van P. Changes in physical therapy providers' use of fall prevention strategies following a multicomponent behavioral change intervention. Phys Ther. 2005; 85:394-403.
34. Leskelä J, Viitanen E, Piirainen A. Client feedback on physiotherapy counselling in primary health care. Patient Education and Counseling. 2005; 56: 218-224.

35. Moseley L. Combined physiotherapy and education is efficacious for chronic low back pain. Australian Journal of Physiotherapy. 2002; 48: 297-302.

36. Alewijnse D, Metsemakers J, Mesters I, Van den Borne B. Effectiveness of pelvic floor muscle exercise therapy supplemented with a health education program to promote long-term adherence among women with urinary incontinence. Neurourology and Urodynamics. 2003; 22:284-295.

37. Molenaara E, van Ameijdena E, Vergouwea Y, Grobbeea D, Numansa M. Effect of nutritional counselling and nutritional plus exercise counselling in overweight adults: A randomized trial in multidisciplinary primary care practice. Family Practice. 2010; 27:143-150.

38. Hay E, Foster N, Thomas E, Peat G, Phelan M, Yates H et al. Effectiveness of community physiotherapy and enhanced pharmacy review for knee pain in people aged over 55 presenting to primary care: pragmatic randomised trial. $\quad$ BMJ. 2005 doi:10.1136/bmj.38977.590752.0B

39. Finkler M, Alvarenga L. Fisioterapia e educação em saúde: investigando um serviço ambulatorial do sus. Boletim da Saúde Porto Alegre. 2005; 19(2):73-82.

40. Talvitie U, Pyöriä O. Discourse analytic study of counseling sessions in stroke physiotherapy. Health Communication. 2006; 20(2): 187-196.

41. Trede, FV. 'Physiotherapists' approaches to low back pain education'. Physiotherapy. 2000; 86(8): 427-433.

42. Miilunpalo S, Laitakari J, Vuori I. Strengths and weaknesses in health counseling in Finnish primary health care. Patient Education and Counseling. 1995; 25: 317-328.

43. Taylor JD, Fletcher JP, Tiarks J. Impact of physical therapist-directed exercise counseling combined with fitness center-based exercise training on muscular strength and exercise capacity in people with type 2 diabetes: a randomized clinical trial. Phys Ther. 2009; 89:884-892.

44. Hollis J, Corden E, Williams P. Longitudinal evaluation of a weight reduction program for patients on peritoneal dialysis. Perit Dial Int. 2005; 25(S3):S152-S154.

45. Miller JS, Litva A, Gabbayb M. Motivating patients with shoulder and back pain to self-care: can a videotape of exercise support physiotherapy? Physiotherapy. 2008; 95: 29-35.

46. Rasinaho $M$, Hirvensalo $M$, Kangas $T$, Leinonen $R$, Lintunen $\mathrm{T}$, Rantanen $\mathrm{T}$. Effect of physical activity counseling on physical activity of older people in Finland. Health Promotion International. 2011. doi:10.1093/heapro/dar057

47. Tsauo J, Yi Lee H, Huei Hsu J, Ying Chen C, Jong Chen C. Physical exercise and health education for neck and shoulder complaints among sedentary workers. J Rehabil Med. 2004; 36: 253-257.

48. Lambeek L, Van Mechelen W, Knol D, Loisel P, Anema J. Randomised controlled trial of integrated care to reduce disability from chronic low back pain in working and 
private life. BMJ. 2010;340:c1035 doi:10.1136/bmj.c1035

49. Friedrich M, Gittler G, Arendasy M, Friedrich K. Longterm effect of a combined exercise and motivational program on the level of disability of patients with chronic low back pain. Spine. 2005; 30(9): 995-1000.

50. Rueda E, Cantos J, Valdivia A, Martínez J. Effectiveness of health education in low back pain in adults. Journal of Sport and Health Research. 2011;3(2):101-12.

51. Kerssens J, Sluijs E, Verhaak P, Knibbe H, Hermans I. Educating patient educators: enhancing instructional effectiveness in physical therapy for low back pain patients. Patient Education and Counseling. 1999; 37: 165-176.

52. World Confederation for Physical Therapy. Physical therapy, physical activity and health. Salud [Internet] 2012 [acceso 4 de marzo de 2015] Disponible en: http://www.wcpt.org/node/33329]

53. World Confederation for Physical Therapy. Disaster management. [Internet] 2016 [acceso 4 de marzo de 2016] Disponible

http://www.wcpt.org/node/33329

54. Glanz K, Rimmer B, Lewis F. Health behavior and health education. Theory, research and practice. San Francisco: Jossey-Bass; 2002.

55. MCkenzie J, Neiger B, Smeltzer J. Planning, implementing, and evaluating health promotion programs. $4^{\text {th }}$ ed. San Francisco: Pearson; 2005.

56. Giguère $A$, Légaré $F$, Grimshaw J, Turcotte $S$, Fiander, $M$, Grudniewicz, A, et al. Printed educational materials: effects on professional practice and healthcare outcomes. Cochrane Database of Systematic Reviews 2012. doi: 10.1002/14651858.CD004398.pub3.

57. Farmer A, Légaré F, Turcot L, Grimshaw J, Harvey E, McGowan J, et al. Printed educational materials: effects on professional practice and health care outcomes. 2008 Cochrane Database of Systematic Reviews. doi:10.1002/14651858.CD004398.pub2.

58. World Health Organization. National Health strategy toolkit. Geneve: World Health Organization and International Telecommunication Union; 2012. 